\title{
Species composition and floristic relationships in southern Goiás forest enclaves
}

\author{
Composição e relações florísticas de encraves florestais no sul de Goiás
}

Paulo Oswaldo Garcia ${ }^{1,2}$, Arthur Sérgio Mouço Valente ${ }^{1}$, Daniel Salgado Pifano ${ }^{1}$, José Felipe Salomão Pessoa ${ }^{3}$, Luiz Carlos Busato ${ }^{4}$, Marco Aurélio Leite Fontes ${ }^{1} \&$ Ary Teixeira Oliveira-Filho ${ }^{5}$

\begin{abstract}
Hinterland fragments of Atlantic forests situated in transitional areas are poorly known with respect to the effects of human impacts on their species composition and regeneration. The purpose of this study was to describe and characterize the structure and composition of the tree community of forest remnants located in Itumbiara, Goiás State, Central Brazil, and to analyze their floristic relationship with other areas of seasonal and transitional vegetation ecotones. Five forest fragments were chosen for tree community sampling. The survey was carried out using PCQ (point-centered quarter) method and 25 points were distributed along linear transects totalling 125 sampling units. Four live trees with circumference at breast height $(1.30 \mathrm{~m}) \geq 15 \mathrm{~cm}$ were recorded at each point. The floristic and phytosociological surveys recorded 149 tree species belonging to 110 genera and 47 families. The analysis of similarity confirmed the ecotonal character with many generalist species and other with occasional occurrence in 'Cerrado' (woody savanna) and seasonal forests. The forest remnants in Itumbiara showed a high tree species diversity. In spite of this, the tree community species suggests higher similarity with savanna vegetation. Key words: Atlantic rainforest, Cerrado, secondary forest, ecotone.

\section{Resumo}

Os fragmentos de Mata Atlântica interioranos localizam-se em região de tensão ecológica e carecem de conhecimento sobre os efeitos de impactos antrópicos sobre sua composição e regeneração. Objetivou-se caracterizar e descrever a estrutura e composição da comunidade arbórea em remanescentes florestais localizados em Itumbiara, Goiás, além de analisar as relações florísticas entre esses e outros fragmentos de formações estacionais e de áreas ecotonais. Foram selecionados cinco fragmentos para a amostragem da comunidade arbórea. Foi aplicado o método de quadrantes, sendo alocados, em cada fragmento, transecções lineares contendo 25 pontos, o que resultou no estabelecimento de 125 unidades amostrais. Em cada ponto, foram inventariados os espécimes arbóreos vivos com circunferência a 1,30 $\mathrm{m}$ acima do solo $\geq 15 \mathrm{~cm}$. As coletas florísticas e o levantamento fitossociológico registraram 149 espécies arbóreas, pertencentes a 110 gêneros e distribuídas em 47 famílias. A análise de similaridade demonstrou o caráter ecotonal, com muitas espécies generalistas e outras de ocorrência mais pontual no Cerrado ou em matas estacionais. Os remanescentes florestais em Itumbiara apresentaram elevada diversidade arbórea. No entanto, a comunidade arbórea sugere relações florísticas mais estreitas com as formações savânicas.
\end{abstract}

Palavras-chave: Mata Atlântica, cerrado, floresta secundária, ecótono.

\section{Introduction}

The Atlantic Domain comprises various threatened ecosystems with high structural and floristic complexity and acknowledged value (SOS Mata Atlântica \& INPE 2008). It covers the mountain ranges of the eastern Brazilian coast and extends far inland (Morellato \& Haddad 2000; Oliveira-Filho \& Fontes 2000), where it intersects with Caatinga to the North, Cerrado in its central part and Araucaria forests to the South (Oliveira-Filho \& Fontes 2000). Remnants are estimated to represent only $7 \%$ of its original extension(SOS Mata Atlântica \& INPE 2008).

\footnotetext{
'Universidade Federal de Lavras, Depto. Ciências Florestais, Lab. Dinâmica de Florestas Tropicais, C.P. 3037, 37200-000, Lavras, MG.

${ }^{2}$ Autor para correspondência: paulogarciamg@posgrad.ufla.br

${ }^{3}$ Universidade Federal de Juiz de Fora, R. José Lourenço Kelmer s.n., 36036-900, Juiz de Fora, MG.

${ }^{4}$ Signus Vitae Comércio e Elaboração de Estudos e Projetos Ambientais Ltda., R. Berlim 73, 27265-000, Volta Redonda, RJ.

${ }^{5}$ Universidade Federal de Minas Gerais, Instituto de Ciências Biológicas, Depto. Botânica, 31270-901, Belo Horizonte, MG.
} 
Deforestation main causes have been lumbering and farming activities, not to mention urban expansion. According to Oliveira-Filho \& Fontes (2000), Atlantic forest formations include the ombrophilous and seasonal types, which are associated to high rainfall and temperatures, and to their seasonality, respectively.

Since agricultural borders, energy production, population and road networks are ever expanding, forest fragmentation provoked by human influence is a continuous process that often has irreparable consequences on the environment (Sabbag 2003). Fragments of Atlantic Forest are scattered throughout what used to be its original territory. In Central Brazil, relict formations are found in southern Goiás and in Mato Grosso do Sul (MMA 2000). Penetration occurs through riparian forests, which act as ecological corridors (Ledru et al. 1998; OliveiraFilho \& Ratter 2000). According to Ab’sáber (2003), these relicts, surrounded and enclaved in morphoclimatic and phytogeographic Cerrado domain, reflect the impact of the Quaternary climatic and paleoecological changes on the formation and fragmentation of corridors or, on a wider scale, on the expansion and retraction of biomes.

In Goiás, the Atlantic Forest, which covers 4\% of the state, includes seasonal deciduous and semideciduous forests (SOS Mata Atlântica \& INPE 2008), associated with recently exposed valleys and slopes with mineral-rich rocks or soils of medium to high fertility (Oliveira-Filho \& Ratter 2000; ImañaEncinas et al. 2007). The township of Itumbiara, southern Goiás, is characterized as an ecotone between Atlantic Forest and Cerrado physiognomies. Since local plant cover is no exception to the national disturbance history, fragments result from the interaction between urban growth and the use of environmental resources. Atlantic Forest, which used to cover approximately $54 \%$ of the township, has now been reduced to 3\% of its surface (SOS Atlantic Forest \& INPE 2008), mainly owing to the excellent farming potential of its soils of basaltic origin (Oliveira-Filho \& Ratter 2000). The region is classified as "insufficiently known, likely to be biologically valuable", so that surveys are needed in order to establish in situ conservation units to protect biological diversity (MMA 2000).

The present study describes the structure and composition of the tree community in forest remnants located in Itumbiara, Goiás, and analyzes their floristic relationships with Atlantic and Cerrado formations to characterize the influence of these domains on species composition. It also analyzes forest regeneration in different-aged communities, in this transitional area, to obtain data that help us understand better the dynamics of the vegetation confronted to the current model of land use and occupation, and make decisions to preserve these ecosystems.

\section{Material and Methods}

This work was conducted at the Fazenda São Fernando (18 $21^{\prime} \mathrm{S}$ and $49^{\circ} 06^{\prime} \mathrm{W}$ ), in Itumbiara, southern Goiás. Located on the banks of river Paranaíba, its circa 1,470 ha are mostly used to grow sugar cane. The landscape presents forest and Cerrado physiognomies, in addition to areas with huge gullies, where research is developed to recover plant communities. Mean altitude is $448 \mathrm{~m}$ and climate is "Aw", according to Köppen's classification, with two distinct seasons: dry winters (4 to 5 months) and humid summers (SEPLAN-GO 2005; EPE 2006). Ninety percent of annual rainfall (between 1,200 and $1,800 \mathrm{~mm}$ ) occur in the wet season, with monthly rates superior to $100 \mathrm{~mm}$ (SEPLAN-GO 2005; EPE 2006). Temperatures have monthly means superior to $18^{\circ} \mathrm{C}$, and annual amplitude is inferior to $4^{\circ} \mathrm{C}$ (EPE 2006). According to Oliveira-Filho \& Fontes (2000), within the Atlantic domain, forest communities located to the North of $23^{\circ} 20^{\prime} \mathrm{S}$, characterized by a dry season superior to 30 days, and at altitudes between 300 and $700 \mathrm{~m}$, are classified as seasonal semi-deciduous submontane forests. The occurrence of various Cerrado physiognomies (Ribeiro \& Walter 1998) was also observed in the region, which includes Cerradão, the forest physiognomy of that Domain. The forest fragments located at the Fazenda São Fernando are usually smaller than 5 ha and at different successional stages.

Based on aerial pictures shot in 1968 (Fig. 1a) and 2005 (Fig. 1b), we selected five fragments to sample the tree community (Fig. 1). In fact, in 1968, two fragments (A1 and A2) were already observed and considered in a more advanced stage of natural regeneration since they presented an established tree community. The three other remnants (R1, R2 and R3) comprehended degraded areas covered mainly with herbaceous and shrub sinusiae, which have been regenerating over the last 40 years and currently present a forest physiognomy. Since the establishment of their tree community is more recent, they were categorized as at an earlier stage of regeneration. The point-centered quarter method (MuellerDombois \& Ellenberg 1974; Brower \& Zar 1984; Martins 1991) was used to describe the tree phytocoenosis. In each fragment, a $10 \mathrm{~m}$ transect 
with 25 equidistant points was established, totaling 5 transects, 125 points and 500 individuals. At each point, four live tree specimens with a circumference at breast level $(\mathrm{CBH})$ equal or superior to $15 \mathrm{~cm}$ were inventoried in 2008. Specimens with multiple stems were only sampled when their quadratic mean $\mathrm{CBH}$ met the minimum criteria for inclusion. The total height, estimated with the help of (10 m long) pruning sheers, and circumference of each individual were recorded. When possible, species were identified in the field. Voucher material of all individuals was collected either to confirm or determine identifications through bibliographic research or comparisons with specimens kept at the ESAL herbarium of the Federal University of Lavras. The family classification of the Angiosperm Phylogeny Group (APG III 2009) was used. Nomenclature was checked based on the Tropicos database (2010), but synonymies and new combinations follow Oliveira-Filho (2009).

For the phytosociological analyses, we separated the samples into two groups: collected in fragments in more advanced stage of regeneration (A1+A2) and gathered in communities at earlier stages (R1+R2+R3). Both Shannon's diversity and Pielou's evenness indexes were obtained. The former were compared both through pairwise t-test (Zar 1999) and diversity curves generated from 500 randomizations of Shannon's index per fragment. The diversity curves were constructed with the help of EstimateS 8.0 (Colwell 2006). To compare the structural patterns between groups, heights and diameters (at $\mathrm{CBH}$ ) were distributed into frequency classes and then compared through a partition chi- square test (Ayres et al. 2007) to quantify the influence of the different disturbance histories on tree species stratification. A Kruskal-Wallis test was run (Zar 1999) to observe possible differences among basal area values in the groups.

The occurrence of the species sampled in this study in different phytophysiognomies of the Atlantic and Cerrado domains was determined according to data found in Treeatlan 1.0 (OliveiraFilho 2009) and Mendonça et al. (1998) and used to construct a Venn diagram to characterize phytogeographical relationships. We also constructed a UPGMA dendrogram based on Sørensen similarity index, involving tree communities from the Cerrado and seasonal forests of centerwestern (Distrito Federal, Goiás and Mato Grosso do Sul) and eastern (Minas Gerais) Brazil (Tab. 1). Data from a preparatory floristic survey carried out in the Fazenda in 2007 were added to the inventory and PC-ORD 4.0 (McCune \& Mefford 1999) was used to construct the dendrogram. An abundance matrix was generated to perform a Detrended Correspondence Analysis (DCA) and verify vegetation gradients between the two groups, due to their distinct preservation history. EstimateS 8.0 also constructed accumulation curves for the specific richness observed (Mao Tau) in the different fragments, after 500 randomizations (Colwell 2006).

\section{Results}

We recorded 149 tree species pertaining to 110 genera and 47 families (Tab. 2), with a predominance of Fabaceae (31 species), which corresponds to
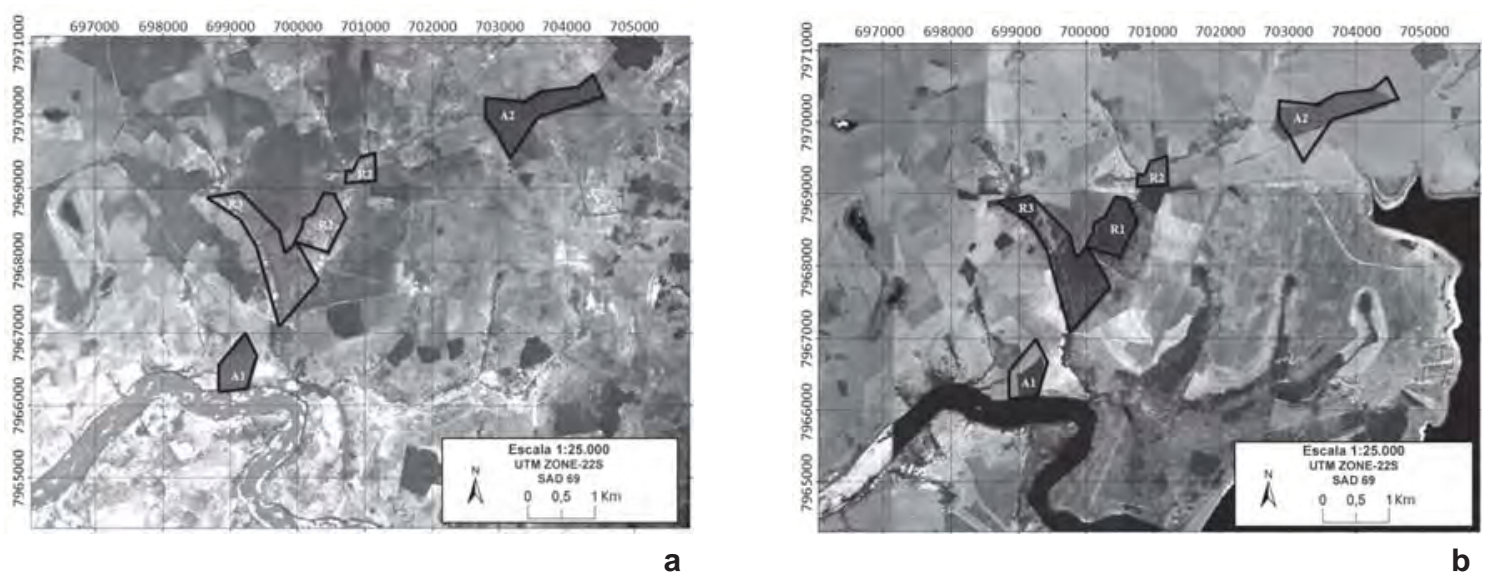

Figure 1- Images taken from the air in 1968 (a) and 2005 (b) of the forest fragments in São Fernando farm, Itumbiara, Goiás State. A1 and A2 are forest fragments in later successional phases; R1, R2 and R3 are forest fragments in earlier successional phases. 
$20.9 \%$ of the richness. Out of these species, $7.3 \%$ were only indentified to the family or genus level. The inventory sampled 500 tree individuals, 300 of which grew in the fragments at an earlier stage of regeneration and 200, in the fragments in a more advanced stage of regeneration, resulting in an estimate of 1,039.5 and 882.2 individuals.ha-1, respectively. As for the tree community, Shannon's index (H') was 4.02 and Pielou's evenness index (J'), 0.87 . The number of sampled species that only presented a single individual was high (32.7\%).

The number of species recorded in the fragments at earlier (Tab. 2) and more advanced stages of regeneration was 72 and 50 species, respectively. The Shannon's index of the fragments at earlier stage, 3.68, was significantly superior ( $\mathrm{t} 0.05$, $430=8.23 ; \mathrm{p}<0.05$ ) to that of the more advanced ones, 3.31. Except for R3, the other fragments at an earlier stage of regeneration presented a Shannon's index superior to that of the fragments in a more advanced stage of regeneration (Fig. 2a), the high value of R1 being noteworthy. All remnants presented a similar specific richness (Fig. 2b), except for R1 whose number of species was quite superior to the others. The species accumulation curves tend to remain constant as the sampling effort increases, suggesting that the species composition sampling was representative (Fig. 2b). All fragments presented similar evenness values: 0.86 in those at an earlier stage of regeneration and 0.84 in the ones at a more advanced stage of regeneration.

Xylopia aromatica (Lam.) Mart., Tapirira guianensis Aubl. and Myracrodruon urundeuva Allemão represented 30\% of the importance value (IV) in the fragments at an earlier stage of regeneration (Tab. 2), but either were not recorded in the fragments in a more advanced stage of regeneration ( $T$. guianensis and M. urundeuva) or had a drastically reduced importance in the community (X. aromatica). Conversely, Nectandra lanceolata Nees, Acacia polyphylla DC. and Trichilia hirta L., which correspond to $31 \%$ of the IV in the fragments in a more advanced stage of regeneration (Tab. 2), presented a reduced importance in the fragments at earlier stages, while $T$. hirta was not even recorded in them. It is worth noting that no species of the genus Trichilia, which mainly formed the understory of the more mature fragments, mainly due to the density of $T$. hirta, were recorded in the fragments at earlier successional stages. No species with higher IVs were common to communities in different stages of regeneration (Tab. 2).
The difference in tree species composition between the fragments in different stages of regeneration became evident in the Detrended Correspondence Analysis (DCA), with high eigenvalues on the first two axes (Braak 1995). Axis 1 (0.879) distinguishes fragments in a more advanced stage of regeneration from those in earlier stage of regeneration (Fig. 3), while axis 2 (0.762) differentiates the composition within the very communities both in advanced and earlier stages (Fig. 3). The biggest differences in structure and composition were observed between fragments A1 and R3, represented at the extremities of axis 1.

With regard to the distribution of the identified species (Fig. 4), 64.2\% are common both to the
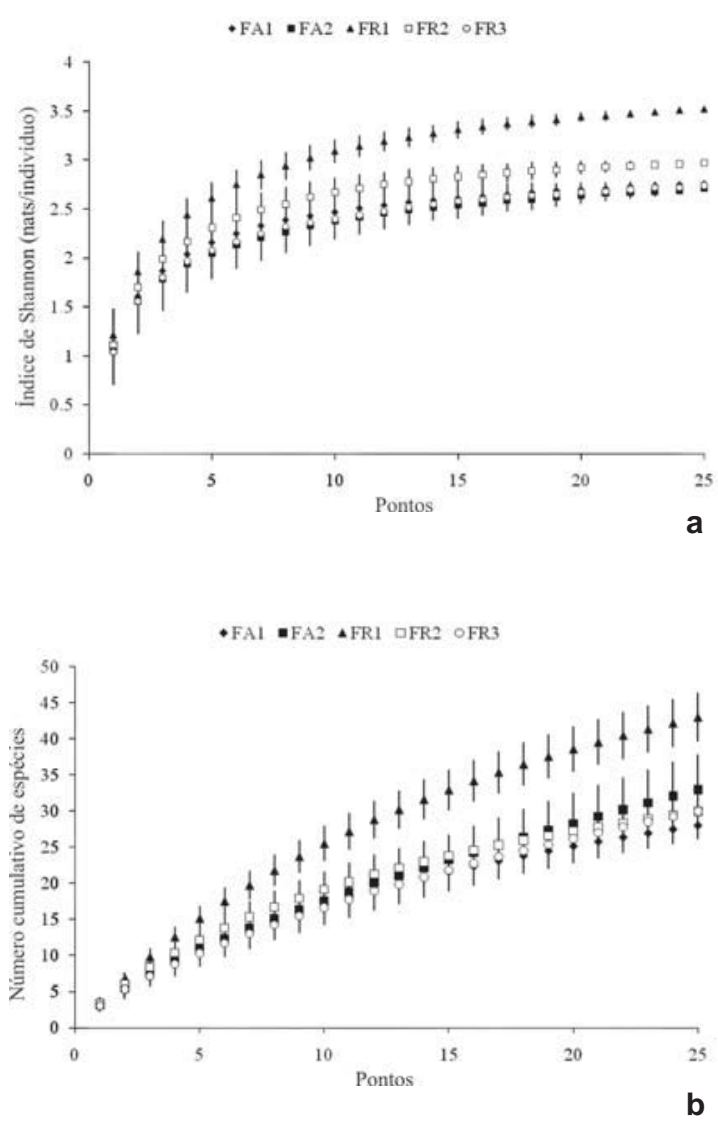

Figure 2-Accumulation curves for the Shannon index (a) and observed number of species (b), obtained from 500 randomized combinations of sample plots used to survey five forest fragments at São Fernando farm, Itumbiara, Goiás State, Brazil. A1 and A2 are forest fragments at later successional phases; R1, R2 and R3 are forest fragments at earlier successional phases. 


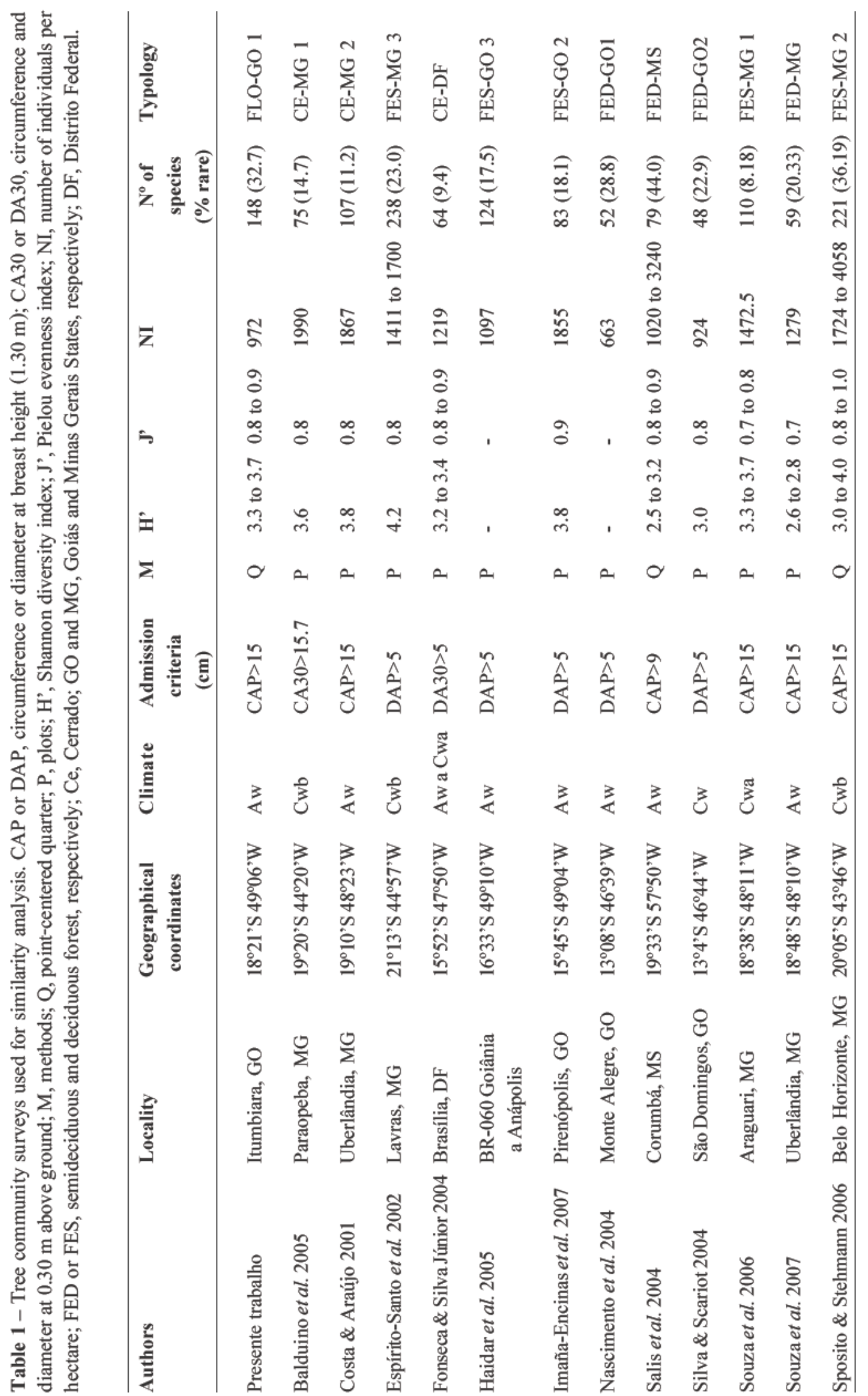


(ombrophilous and/or seasonal) forest and savanna physiognomies. The species that only occur in the Atlantic Domain accounted for 33.5\% of the total. Nonetheless, the floristic relationships between the tree community of the Fazenda São Fernando and other formations point out a greater similarity to Cerrado vegetations (Fig. 5).

Analyzing the frequency distribution of individuals in height $\left(\mathrm{X}^{2} 0.05,5=30.63 ; \mathrm{p}<0.0001\right)$ and diameter $\left(\mathrm{X}^{2} 0.05,5=24.72 ; \mathrm{p}=0.01\right)$ classes showed significant differences between fragments. A reduction of higher specimens was observed in the fragments at earlier stages of regeneration, especially of individuals higher than $12 \mathrm{~m}$ (Fig. 6a) and over $25 \mathrm{~cm}$ in diameter (Fig. 6b). On the whole, individuals are between $4.1 \mathrm{~m}$ and $8 \mathrm{~m}$ high and correspond to $48 \%$ in the earlier fragments and $54.6 \%$ in the more advanced ones. The distribution of individuals in diameter classes (Fig. 6b) showed a higher abundance of sampled trees in the smaller size classes and a gradual decrease as we move toward bigger classes. A difference (H0.05, 1 = 7.02; $\mathrm{p}=0.008$ ) between basal area values was also observed: they were higher in the more mature fragments, $23.71 \mathrm{~m}^{2}$.ha-1, than in the ones at an earlier stage of regeneration, $11.11 \mathrm{~m}^{2} \cdot \mathrm{ha}^{-1}$.

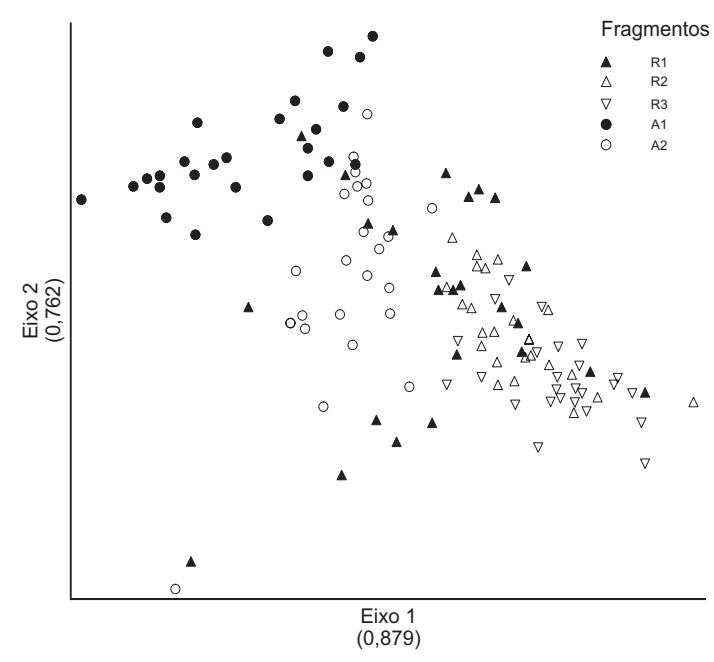

Figure 3 - Detrended correspondence analysis (DCA) of 125 sample points used to survey the forest fragments at São Fernando farm, Itumbiara, GO. A1 and $\mathrm{A} 2$ are forest fragments in later successional phases; R1, R2 and R3 are forest fragments in earlier successional phases.

\section{Discussion}

This survey recorded generalist species with a wide geographical distribution that colonize from Atlantic rainforest to hinterland savanna communities, as is the case of Amaioua guianensis Aubl., Apuleia leiocarpa (Vogel) J.F. Macbr., Casearia sylvestris Sw. and Cecropia pachystachya Trécul (Oliveira-Filho \& Fontes 2000; Oliveira-Filho 2009). On the other hand, Byrsonima crassa Nied., Ferdinandusa ovalis Pohl, Mouriri elliptica Mart., Sorocea sprucei (Baill.) J.F. Macbr., Tachigali vulgaris L.G. Silva \& H.C. Lima - predominantly distributed in Central Brazil - and Erythroxylum tortuosum Mart., Salacia crassifolia (Mart.) G.Don and Zeyheria montana Mart., characteristic of Cerrado (Mendonça et al. 1998; Oliveira-Filho 2009), were also reported. According to Mendonça et al. (1998), the distribution of F. ovalis and S. sprucei in the Cerrado Domain is limited to riparian forests, while B. crassa, M. elliptica and T. vulgaris are more directly associated with lower savanna formations. On the other hand, among the species predominantly distributed in the Atlantic Domain “sensu latissimo" (Oliveira-Filho 2006), we reported Machaerium stipitatum (DC.) Vogel, Nectandra lanceolata Nees, Bauhinia ungulata L., Manihot

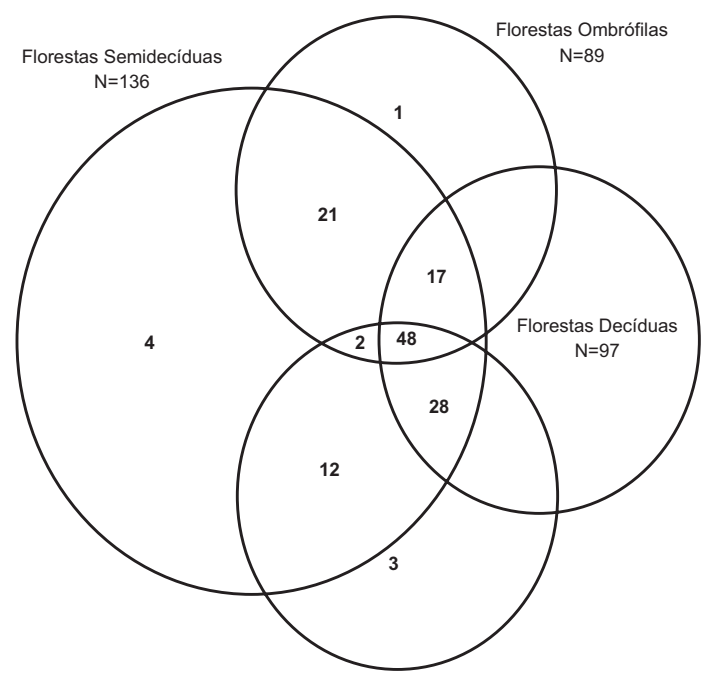

Figure 4 - Venn diagram produced for the tree species surveyed in forest fragments of the São Fernando farm, Itumbiara, Goiás State, floristic and phytossociological stands showing number of species shared by rain, semideciduous and deciduous forests and Cerrado (woody savanna) vegetation. $\mathrm{N}$, total number of species. 
Table 2 - Families and species recorded in floristic and phytossociological surveys carried out in forest fragments with different disturbance histories in Itumbiara, Goiás State. N, number of individuals; AB, basal area in $\mathrm{m}^{2}$; NP, number of point-quarter species occurrence; VI, Importance Value; ***Species surveyed only in floristic surveys.

\begin{tabular}{|c|c|c|c|c|c|c|c|c|}
\hline \multirow[t]{3}{*}{ FAMILIES/SPECIES } & \multicolumn{8}{|c|}{ Floristic communities } \\
\hline & \multicolumn{4}{|c|}{ Initial stage } & \multicolumn{3}{|c|}{ Advanced stage } & \multirow[b]{2}{*}{ VI } \\
\hline & $\mathbf{N}$ & $\mathbf{A B}$ & NP & VI & $\mathbf{N}$ & AB & NP & \\
\hline \multicolumn{9}{|l|}{ Anacardiaceae } \\
\hline Astronium fraxinifolium Schott. ex Spreng.*** & - & - & - & - & - & - & - & - \\
\hline Myracrodruon urundeuva Allemão & - & - & - & - & 22 & 0.219 & 17 & 21.00 \\
\hline Tapirira guianensis Aubl. & - & - & - & - & 26 & 0.421 & 18 & 29.03 \\
\hline Thyrsodium spruceanum Salzm. \& Benth. & - & - & - & - & 1 & 0.002 & 1 & 0.79 \\
\hline \multicolumn{9}{|l|}{ Annonaceae } \\
\hline Annona crassiflora Mart. & - & - & - & - & 3 & 0.024 & 3 & 2.95 \\
\hline Cardiopetalum calophyllum Schltdl.*** & - & - & - & - & - & - & - & - \\
\hline Guatteria aff. pogonopus Mart.*** & - & - & - & - & - & - & - & - \\
\hline Xylopia aromatica (Lam.) Mart. & 2 & 0.020 & 2 & 2,62 & 43 & 0.332 & 30 & 36.73 \\
\hline Xylopia sericea A.St.-Hil.*** & - & - & - & - & - & - & - & - \\
\hline \multicolumn{9}{|l|}{ Apocynaceae } \\
\hline Aspidosperma cf. cuspa (Kunth) S.F.Blake ex Pittier*** & - & - & - & - & - & - & - & - \\
\hline Aspidosperma cylindrocarpon Müll. Arg. & 7 & 0.087 & 6 & 8.85 & 6 & 0.079 & 5 & 6.46 \\
\hline Aspidosperma parvifolium A. DC. & - & - & - & - & 2 & 0.009 & 2 & 1.74 \\
\hline \multicolumn{9}{|l|}{ Araliaceae } \\
\hline Schefflera macrocarpa (Cham. \& Schltdl.) Frodin & 1 & 0.006 & 1 & 1.23 & 1 & 0.003 & 1 & 0.83 \\
\hline Schefflera morototoni (Aubl.) Maguire et al.*** & - & - & - & - & - & - & - & - \\
\hline \multicolumn{9}{|l|}{ Arecaceae } \\
\hline Mauritia flexuosa L.f.*** & - & - & - & - & - & - & - & - \\
\hline \multicolumn{9}{|l|}{ Bignoniaceae } \\
\hline Cybistax antisyphilitica (Mart.) Mart. & 1 & 0.005 & 1 & 1.21 & - & - & - & - \\
\hline Tabebuia chrysotricha (Mart. ex A.DC.) Standl.*** & - & - & - & - & - & - & - & - \\
\hline Tabebuia ochracea (Cham.) Standl. & - & - & - & - & 1 & 0.003 & 1 & 0.83 \\
\hline Tabebuia sp. & - & - & - & - & 1 & 0.004 & 1 & 0.85 \\
\hline Zeyheria montana Mart.*** & - & - & - & - & - & - & - & - \\
\hline \multicolumn{9}{|l|}{ Boraginaceae } \\
\hline Cordia ecalyculata Vell. & 1 & 0.003 & 1 & 1.18 & - & - & - & - \\
\hline \multicolumn{9}{|l|}{ Burseraceae } \\
\hline Protium heptaphyllum (Aubl.) Marchand & 3 & 0.106 & 3 & 5.33 & 3 & 0.033 & 3 & 3.24 \\
\hline \multicolumn{9}{|l|}{ Calophyllaceae } \\
\hline Kielmeyera sp. & 1 & 0.005 & 1 & 1.21 & - & - & - & - \\
\hline \multicolumn{9}{|l|}{ Celastraceae } \\
\hline Maytenus ilicifolia (Schrad.) Planch. & 2 & 0.010 & 2 & 2.43 & - & - & - & - \\
\hline Salacia crassifolia (Mart.) G. Don & 5 & 0.033 & 5 & 6.22 & - & - & - & - \\
\hline \multicolumn{9}{|l|}{ Chrysobalanaceae } \\
\hline Hirtella glandulosa Spreng. & - & - & - & - & 1 & 0.015 & 1 & 1.20 \\
\hline Hirtella hebeclada Moric. ex DC. & 3 & 0.049 & 3 & 4.27 & - & - & - & - \\
\hline Hirtella racemosa Lam.*** & - & - & - & - & - & - & - & - \\
\hline Hirtella sp. & - & - & - & - & 3 & 0.020 & 2 & 2.42 \\
\hline Licania kunthiana Hook. f. & 8 & 0.468 & 7 & 17.06 & - & - & - & - \\
\hline \multicolumn{9}{|l|}{ Clusiaceae } \\
\hline Symphonia globulifera L.f.*** & - & - & - & - & - & - & - & - \\
\hline Combretaceae & & & & & & & & \\
\hline Terminalia argentea (Cambess.) Mart.*** & - & - & - & - & - & - & - & - \\
\hline Terminalia fagifolia Mart. & 1 & 0.002 & 1 & 1.16 & 2 & 0.006 & 1 & 1.26 \\
\hline Connaraceae & & & & & & & & \\
\hline Rourea cf. induta Planch. & - & - & - & - & 1 & 0.005 & 1 & 0.88 \\
\hline Dilleniaceae & & & & & & & & \\
\hline Curatella americana L. & - & - & - & - & 10 & 0.159 & 9 & 11.89 \\
\hline Ebenaceae & & & & & & & & \\
\hline Diospyros hispida A.DC.*** & - & - & - & - & - & - & - & - \\
\hline
\end{tabular}


FAMILIES/SPECIES
Floristic communities

Initial stage

N AB NP

\section{Erythroxylaceae}

Erythroxylum deciduum A. St.-Hil.

Erythroxylum tortuosum Mart.

Euphorbiaceae

Manihot anomala Pohl

Maprounea guianensis Aubl.

Pera glabrata (Schott) Poepp. ex Baill.

Sapium glandulatum (L.) Morong

\section{Fabaceae}

Acacia polyphylla DC.

Acosmium dasycarpum (Vogel) Yakovlev

Albizia niopoides (Spruce ex Benth.) Burkart***

Anadenanthera colubrina (Vell.) Brenan

Apuleia leiocarpa (Vogel) J.F.Macbr.***

Bauhinia brevipes Vogel

Bauhinia rufa (Bong.) Steud.

Bauhinia ungulata L.***

Bowdichia virgilioides Kunth

Copaifera langsdorffii Desf.

Deguelia costata (Benth.) Az.-Tozzi

Dimorphandra mollis Benth.

Dipteryx alata Vogel

Diptychandra aurantiaca Tul.***

Enterolobium gummiferum (Mart.) J.F.Macbr.***

Hymenaea martiana Hayne***

Hymenaea stigonocarpa Mart. ex Hayne

Inga laurina (Sw.) Willd.***

Inga sessilis (Vell.) Mart.

Machaerium acutifolium Vogel ${ }^{* * *}$

Machaerium opacum Vogel

Machaerium stipitatum (DC.) Vogel

Mimosa bimucronata (DC.) Kuntze***

Ormosia fastigiata Tul.

Plathymenia reticulata Benth.

Platypodium elegans Vogel

Pterodon emarginatus Vogel

Stryphnodendron adstringens (Mart.) Cov.

Tachigali subvelutina (Benth.) Oliveira-Filho

Tachigali vulgaris L.G.Silva \& H.C.Lima***

Vatairea macrocarpa (Benth.) Ducke***

Humiriaceae

Sacoglottis cf. guianensis Benth.

\section{Icacinaceae}

Emmotum nitens (Benth.) Miers

Indeterminada

Indeterminada

\section{Lauraceae}

Nectandra cuspidata Nees***

Nectandra lanceolata Nees

Ocotea cf. corymbosa (Meisn.) Mez***

\section{Lecythidaceae}

Cariniana legalis (Mart.) Kuntze

Lecythis sp.

\section{Loganiaceae}

Antonia ovata Pohl***

Strychnos cf. pseudoquina A.St.-Hil.***

Malpighiaceae

Byrsonima cf. intermedia A.Juss.***

Advanced stage

AB NP VI

$\begin{array}{lllll}- & 8 & 0.069 & 7 & 7.63 \\ - & 1 & 0.016 & 1 & 1.23\end{array}$

0.0101

$\begin{array}{ll}- & - \\ 0.003 & 1\end{array}$

$\begin{array}{lll}1 & 0.017 \quad 2\end{array}$

$1.31-$

$1.17 \quad 5$

$\begin{array}{lll}0.008 & 1 & 0.97\end{array}$

3.06

$0.271 \quad 13$

22.111

$\begin{array}{lll}0.017 & 1 & 1.26\end{array}$

-

1.3023

-

0.009

-

$-$

-

-

$0.076 \quad 1$

0.0121

0.0715

0.0072

- 1

$\begin{array}{lll}0.004 & 1 & 0.85\end{array}$

$27.58-$

$-$

$\begin{array}{lll}0.007 & 2 & 1.68\end{array}$

$0.029 \quad 1 \quad 1.63$

0.0201159

$\begin{array}{lll}0.113 & 3 & 5.72\end{array}$

$\begin{array}{lll}0.002 & 1 & 0.80\end{array}$

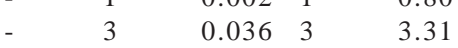

1.29

- $\quad-\quad-$

0.0243

0.0021

$\begin{array}{lll}0.019 & 3 & 2.80\end{array}$

$\begin{array}{lll}0.081 & 1 & 3.59\end{array}$

$0.103 \quad 3 \quad 5.42$

$\begin{array}{lll}0.103 & 3 & 5.42 \\ 0.020 & 1 & 1.35\end{array}$

$\begin{array}{lll}0.013 \quad 2 & 1.87\end{array}$

- - -

$\begin{array}{lll}0.221 & 8 & 13.11\end{array}$

$0.026 \quad 6 \quad 5.21$

2.876

$-$

0.0142

1.89

$0.016 \quad 1$

$0.018 \quad 1$

1.45

$34 \quad 0.717 \quad 20$

$42.77 \quad 1$

0.0021

0.79 


\section{FAMILIES/SPECIES}

Floristic communities

\begin{tabular}{|c|c|c|c|c|c|c|c|c|}
\hline & \multicolumn{3}{|c|}{ Initial stage } & \multicolumn{5}{|c|}{ Advanced stage } \\
\hline & $\mathbf{N}$ & AB & NP & VI & $\mathbf{N}$ & AB & NP & VI \\
\hline Byrsonima cf. verbascifolia (L.) DC.*** & - & - & - & - & - & - & - & - \\
\hline Byrsonima coccolobifolia Kunth. & - & - & - & - & 2 & 0.020 & 1 & 1.68 \\
\hline Byrsonima crassa Nied. & - & - & - & - & 1 & 0.006 & 1 & 0.92 \\
\hline Byrsonima sericea DC. & - & - & - & - & 6 & 0.032 & 5 & 5.01 \\
\hline Byrsonima sp. & 2 & 0.239 & 2 & 6.68 & - & - & - & - \\
\hline \multicolumn{9}{|l|}{ Malvaceae } \\
\hline Apeiba tibourbou Aubl.*** & - & - & - & - & - & - & - & - \\
\hline Ceiba speciosa (A.St.-Hil.) Ravenna*** & - & - & - & - & - & - & - & - \\
\hline Guazuma ulmifolia Lam. & 4 & 0.019 & 3 & 4.21 & 3 & 0.054 & 2 & 3.47 \\
\hline Luehea grandiflora Mart. \& Zucc. & 3 & 0.067 & 3 & 4.60 & 10 & 0.033 & 6 & 6.79 \\
\hline Pseudobombax longiflorum (Mart. \& Zucc.) A.Robyns*** & - & - & - & - & - & - & - & - \\
\hline \multicolumn{9}{|l|}{ Melastomataceae } \\
\hline Miconia albicans (Sw.) Triana & - & - & - & - & 2 & 0.007 & 2 & 1.69 \\
\hline Miconia sp. & 1 & 0.002 & 1 & 1.17 & - & - & - & - \\
\hline Mouriri elliptica Mart.*** & - & - & - & - & - & - & - & - \\
\hline \multicolumn{9}{|l|}{ Meliaceae } \\
\hline Guarea guidonia (L.) Sleumer & 1 & 0.007 & 1 & 1.25 & - & - & - & - \\
\hline Guarea macrophylla Vahl*** & - & - & - & - & - & - & - & - \\
\hline Trichilia claussenii C.DC. & 8 & 0.056 & 6 & 8.76 & - & - & - & - \\
\hline Trichilia hirta L. & 21 & 0.108 & 13 & 20.58 & - & - & - & - \\
\hline Trichilia pallida Sw. & 3 & 0.024 & 3 & 3.80 & - & - & - & - \\
\hline \multicolumn{9}{|l|}{ Moraceae } \\
\hline Brosimum gaudichaudii Trécul*** & - & - & - & - & - & - & - & - \\
\hline Sorocea sprucei (Baill.) J.F.Macbr. & 1 & 0.007 & 1 & 1.26 & - & - & - & - \\
\hline \multicolumn{9}{|l|}{ Myristicaceae } \\
\hline Virola sebifera Aubl. & 6 & 0.065 & 5 & 7.31 & 8 & 0.030 & 6 & 6.02 \\
\hline \multicolumn{9}{|l|}{ Myrtaceae } \\
\hline Campomanesia sp. & - & - & - & - & 3 & 0.011 & 3 & 2.54 \\
\hline Eugenia adenantha O.Berg*** & - & - & - & - & - & - & - & - \\
\hline Eugenia brasiliensis Lam. & 1 & 0.005 & 1 & 1.21 & - & - & - & - \\
\hline Eugenia punicifolia (Kunth) DC.*** & - & - & - & - & - & - & - & - \\
\hline Myrcia sp. & - & - & - & - & 1 & 0.006 & 1 & 0.92 \\
\hline Myrcia tomentosa (Aubl.) DC.*** & - & - & - & - & - & - & - & - \\
\hline Myrtaceae sp.1 & 3 & 0.050 & 3 & 4.30 & - & - & - & - \\
\hline Psidium salutare (Kunth) O.Berg & - & - & - & - & 8 & 0.085 & 7 & 8.12 \\
\hline Siphoneugena cf. densiflora O.Berg*** & - & - & - & - & - & - & - & - \\
\hline \multicolumn{9}{|l|}{ Ochnaceae } \\
\hline Ouratea hexasperma (A.St.-Hil.) Baill. & 1 & 0.004 & 1 & 1.20 & - & - & - & - \\
\hline \multicolumn{9}{|l|}{ Olacaceae } \\
\hline Heisteria silvianii Schwacke & - & - & - & - & 2 & 0.005 & 2 & 1.62 \\
\hline Ximenia americana $\mathrm{L}$. & - & - & - & - & 2 & 0.007 & 2 & 1.69 \\
\hline \multicolumn{9}{|l|}{ Polygonaceae } \\
\hline Coccoloba mollis Casar. & - & - & - & - & 4 & 0.043 & 4 & 4.29 \\
\hline \multicolumn{9}{|l|}{ Primulaceae } \\
\hline Myrsine umbellata Mart. & 1 & 0.007 & 1 & 1.25 & 4 & 0.015 & 2 & 2.59 \\
\hline \multicolumn{9}{|l|}{ Proteaceae } \\
\hline Roupala montana Aubl. & - & - & - & - & 1 & 0.003 & 1 & 0.82 \\
\hline \multicolumn{9}{|l|}{ Rubiaceae } \\
\hline Alibertia edulis (L.C.Rich.) A.Rich. ex DC. & - & - & - & - & 2 & 0.010 & 2 & 1.79 \\
\hline Amaioua guianensis Aubl. & 1 & 0.004 & 1 & 1.19 & - & - & - & - \\
\hline Cordiera concolor (Cham.) Kuntze & 2 & 0.008 & 2 & 2.40 & - & - & - & - \\
\hline Ferdinandusa ovalis Pohl & - & - & - & - & 4 & 0.047 & 4 & 4.39 \\
\hline Guettarda uruguensis Cham. \& Schltdl. & - & - & - & - & 1 & 0.008 & 1 & 0.99 \\
\hline Ixora brevifolia Benth. & 5 & 0.069 & 5 & 6.89 & - & - & - & - \\
\hline Simira sampaioana (Standl.) Steyerm.*** & - & - & - & - & - & - & - & - \\
\hline \multicolumn{9}{|l|}{ Rutaceae } \\
\hline Zanthoxylum rhoifolium Lam. & - & - & - & - & 3 & 0.102 & 3 & 5.38 \\
\hline
\end{tabular}

Rodriguésia 62(1): 123-137. 2011 


\begin{tabular}{|c|c|c|c|c|c|c|c|c|}
\hline \multirow[t]{3}{*}{ FAMILIES/SPECIES } & \multicolumn{8}{|c|}{ Floristic communities } \\
\hline & \multirow[b]{2}{*}{$\mathbf{N}$} & \multicolumn{3}{|c|}{ Initial stage } & \multicolumn{3}{|c|}{ Advanced stage } & \multirow[b]{2}{*}{ VI } \\
\hline & & AB & NP & VI & $\mathbf{N}$ & AB & NP & \\
\hline \multicolumn{9}{|l|}{ Salicaceae } \\
\hline Casearia sylvestris Sw. & 7 & 0.033 & 6 & 7.84 & - & - & - & - \\
\hline \multicolumn{9}{|l|}{ Sapindaceae } \\
\hline Cupania racemosa (Vell.) Radlk. & - & - & - & - & 6 & 0.034 & 6 & 5.48 \\
\hline Cupania vernalis Cambess. & - & - & - & - & 2 & 0.008 & 2 & 1.73 \\
\hline Dilodendron bipinnatum Radlk.*** & - & - & - & - & - & - & - & - \\
\hline Magonia pubescens A.St.-Hil. & - & - & - & - & 1 & 0.007 & 1 & 0.95 \\
\hline Matayba elaeagnoides Radlk. & - & - & - & - & 2 & 0.015 & 2 & 1.93 \\
\hline Matayba guianensis Aubl. & 3 & 0.128 & 2 & 5.11 & 8 & 0.043 & 7 & 6.83 \\
\hline \multicolumn{9}{|l|}{ Sapotaceae } \\
\hline Chrysophyllum sp. & - & - & - & - & 6 & 0.061 & 6 & 6.31 \\
\hline Pouteria ramiflora (Mart.) Radlk. & - & - & - & - & 1 & 0.006 & 1 & 0.92 \\
\hline Pouteria torta (Mart.) Radlk.*** & - & - & - & - & - & - & - & - \\
\hline \multicolumn{9}{|l|}{ Siparunaceae } \\
\hline Siparuna guianensis Aubl. & 9 & 0.034 & 9 & 10.72 & 4 & 0.014 & 4 & 3.37 \\
\hline \multicolumn{9}{|l|}{ Solanaceae } \\
\hline Solanum lycocarpum A.St.-Hil.*** & - & - & - & - & - & - & - & - \\
\hline \multicolumn{9}{|l|}{ Styracaceae } \\
\hline Styrax ferrugineus Nees \& Mart. & 2 & 0.021 & 1 & 2.01 & 1 & 0.003 & 1 & 0.82 \\
\hline Styrax pohlii A.DC.*** & - & - & - & - & - & - & - & - \\
\hline \multicolumn{9}{|l|}{ Urticaceae } \\
\hline Cecropia pachystachya Trécul & - & - & - & - & 5 & 0.034 & 4 & 4.33 \\
\hline \multicolumn{9}{|l|}{ Vochysiaceae } \\
\hline Callisthene major Mart. & 1 & 0.008 & 1 & 1.26 & 1 & 0.191 & 1 & 6.69 \\
\hline Qualea cf. parviflora Mart. & - & - & - & - & 1 & 0.009 & 1 & 1.01 \\
\hline Qualea grandiflora Mart.*** & - & - & - & - & - & - & - & - \\
\hline Qualea multiflora Mart. & 2 & 0.018 & 1 & 1.95 & 4 & 0.012 & 3 & 2.91 \\
\hline Vochysia acuminata Bong.*** & - & - & - & - & - & - & - & - \\
\hline Vochysia cf. pyramidalis Mart.*** & - & - & - & - & - & - & - & - \\
\hline Vochysia thyrsoidea Pohl & 1 & 0.008 & 1 & 1.26 & - & - & - & - \\
\hline
\end{tabular}

anomala Pohl and Trichilia hirta L., and B. ungulata, M. anomala and T. hirta, which are related to seasonal forests (Oliveira-Filho 2009). Other species found in ecotonal areas as Dipterix alata Vogel, Emmotum nitens (Benth.) Miers, Hirtella glandulosa Spreng., Magonia pubescens A. St.-Hil. and Myracrodruon urundeuva Allemão reaffirm the transition between Atlantic Forest and Cerrado Domain (Oliveira-Filho \& Ratter 2002). Few recorded species are exclusive to semi-deciduous formations, as Ixora brevifolia Benth. and Styrax pohlii A. DC. (Oliveira-Filho 2009). Among the species associated with the semi-deciduous forests of western Brazil (Oliveira-Filho \& Fontes 2000) are Acosmium dasycarpum (Vogel) Yakovlev, Albizia niopoides (Spruce ex Benth.) Burkart, Apeiba tibourbou Aubl., Astronium fraxinifolium Schott. ex Spreng., Callisthene major Mart., Cordiera concolor (Cham.) Kuntze, Diospyros hispida A.DC., Eugenia punicifolia (Kunth) DC., Machaerium acutifolium
Vogel, Myrcia tomentosa (Aubl.) DC., Platypodium elegans Vogel, Siparuna guianensis Aubl., Siphoneugena cf. densiflora O.Berg, Terminalia argentea (Cambess.) Mart., Virola sebifera Aubl., Xylopia aromatica (Lam.) Mart. and Zanthoxylum rhoifolium Lam.

The high values of specific richness and diversity reported for the tree phytocoenosis of the Fazenda São Fernando were compatible with those obtained in semi-deciduous forests in the Atlantic Domain (Meira-Neto \& Martins 2002) and are also characteristic of southern Goiás woodlands, which are considered as ecotonal areas (OliveiraFilho \& Ratter 2002). Central Brazil forests constitute an important link between northeastern, southeastern and Pantanal seasonal formations and pre-Amazonian vegetation (Oliveira-Filho \& Ratter 2000; Felfili 2003; Haidar et al. 2005) and, naturally, their composition is influenced by these zones (Leitão Filho 1987). According to surveys carried out by Haidar et al. 
(2005) and Imaña-Encinas et al. (2007) in Goiás semi-deciduous forests, the most representative species in the community structures were Acacia polyphylla DC., Amaioua guianensis Aubl., Anadenanthera colubrina (Vell.) Brenan, Callisthene major Mart., Dilodendron bipinnatum Radlk., Guazuma ulmifolia Lam., Myracrodruon urundeuva Allemão, Protium heptaphyllum (Aubl.) Marchand, Qualea multiflora Mart. and Tapirira guianensis Aubl., which were also recorded in the present survey. Nonetheless, the similarity of the tree community inventoried in Itumbiara to savanna formations reveals the remarkable influence of the Cerrado Domain, highlighted by the increased importance of genera associated with it, as Byrsonima and Qualea (Ledru 1993; Mendonça et al. 1998; Oliveira-Filho \& Ratter 2002; Ratter et al. 2003; Carvalho \& Marques-Alves 2008). Thus, the records of species characteristic of Goiás semi-deciduous forests and of species distributed in the Atlantic Domain as well as the floristic relationships to Cerrado manifest that this area is an ecotone between seasonal and Cerradão types. Delimiting the extension of Cerradão communities (Cerrado Domain) and of seasonal forests (Atlantic Forest) in transition areas is complex (Ribeiro et al. 1983). Cerradão can act as a distribution limit for tree species of the Atlantic Domain (Costa \& Araújo 2001) and share a high number of species with the latter (Rizzini 1979).

Differences among the compositions of plant communities can derive from habitat heterogeneity, climatic and edaphic conditions and modifications, inter-specific interactions, and disturbance histories (Whittaker 1972; Ledru 1993; Oliveira-Filho et al. 2001; Tabarelli et al. 2004; Sposito \& Stehmann 2006). Since they modify the demographic rates, the geographical distribution of plant populations and impact the extension of the biome zones (Ledru 1993; Ledru et al. 1996; Ledru et al. 1998; Hill \& Curran 2003; Tabarelli et al. 2004; Durigan \& Ratter 2006; Malhi et al. 2008; Lenoir et al. 2008; Ledru et al. 2009; Mantgem et al. 2009), such factors interfere in the vegetation resilience and generate floristic dissimilarities. According to Oliveira-Filho \& Ratter (2002), the forest physiognomies of Central Brazil can be distinguished according to the availability of water in the soil and fertility of the latter. Then, except for the variable climate (the proximity between fragments leads us to presuppose they are submitted to similar climatic conditions), the other factors potentially affect the floristic

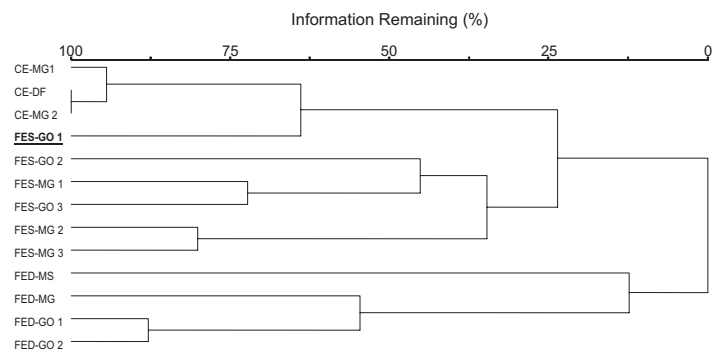

Figure 5 - Floristic relationships obtained from Sorensen similarity index including the present study and the other surveys listed in Table 1.

composition of the fragments at the Fazenda São Fernando. There was a clear distinction between species whose high importance values stood out, as Xylopia aromatica and Nectandra lanceolata, which demonstrated a lower recruitment capacity in forests in more advanced and earlier successional stages, respectively. Floristic separations also occurred between fragments of similar ages, albeit on a minor scale. Again, the composition peculiarities between fragments can reflect soil modifications (Oliveira-Filho \& Ratter 2002) or correspond to distinct successional stages (Durigan \& Ratter 2006). Also, the differences between fragments of the Fazenda São Fernando can be explained by past disturbances, since those in earlier stages of regeneration were exposed to human activity for a longer period of time. Such disturbances affect both water sources and the availability of propagules to restore vegetation (Castellani \& Stubblebine 1993; Roberts \& Gillian 1995; Frelich et al. 1998).

The tree density obtained for the community inventoried in Itumbiara is inferior to that found in savanna formations and other semi-deciduous forests, and is closer to that of Goiás deciduous forests (Tab. 1). Lower densities can be attributed to the presence of clearings (caused by the fall of trees or parts of them) and/or to the penetration of Cerrado physiognomies, characterized by fewer and lower tree specimens, into the forest. In such circumstances, the lesser presence of trees entails an increase in the mean value of the point-plant distance and, consequently, in the mean area, which, in turn, implies a lower estimate of absolute density. In the fragments in more advanced stages of regeneration, we mainly observed clearings, while in those at earlier stages of regeneration we found intersections with lower savanna types. In the communities regenerated 

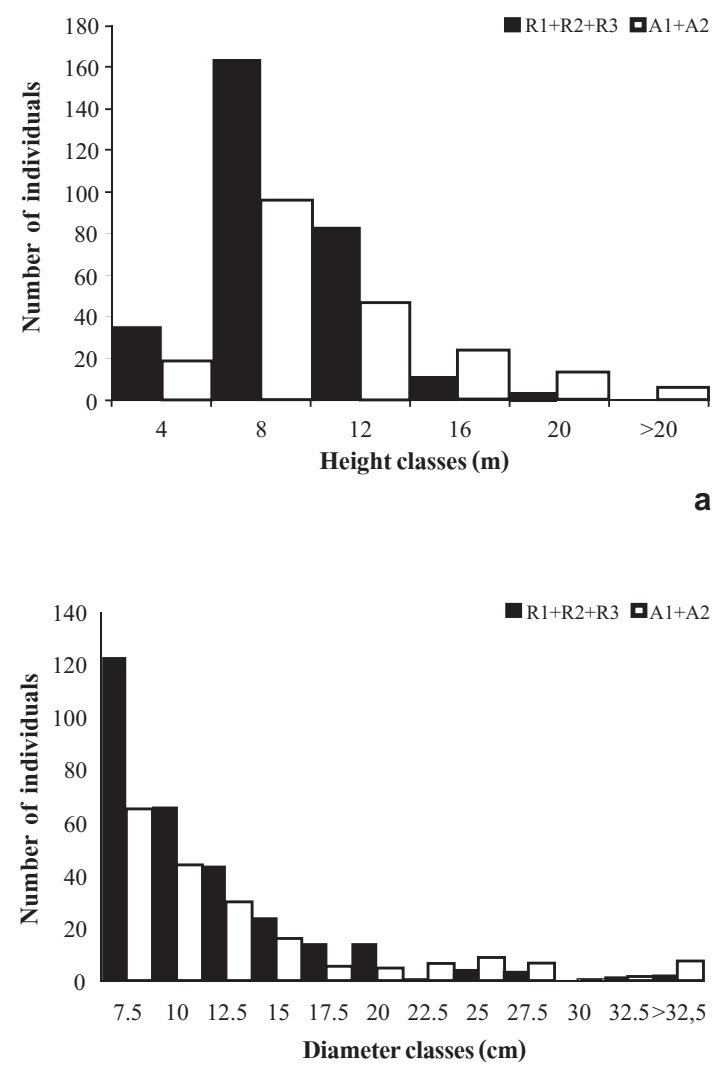

b

Figure 6 - Frequency distribution into height (A) and diameter (B) classes of the tree specimens surveyed in São Fernando farm forest fragments, Itumbiara, Goiás State. A1+A2, forest fragments in later successional phases; R1+R2+R3, forest fragments in earlier successional phases

over the last 40 years, basal area and structural complexity were lower. According to Rosa \& Schiavini (2006), the tree stratum of Central Brazil mesophilic forests comprises individuals between 15 and $25 \mathrm{~m}$ high. Yet, mainly in the fragments in earlier stages of regeneration, we checked the absence of specimens higher than $12 \mathrm{~m}$, which made these communities similar to Cerradão or Campo Cerrado phytophysiognomies, depending on the fragments (according to Silva \& Bates 2002; Durigan \& Ratter 2006). In this survey, biomass loss and stratum reductions in the canopy are probably due to the fact that sampling included Cerrado patches composed of lower specimens. It is worth highlighting that in the more mature fragments, where intersections with lower savanna communities were less frequent, the basal area value $\left(23.71 \mathrm{~m}^{2} . \mathrm{ha}^{-1}\right)$ was superior to that obtained by Haidar et al. (2005) (19,9 m².ha' $\left.{ }^{-1}\right)$ and Imaña-Encinas et al. (2007) (20.08 $\left.\mathrm{m}^{2} \cdot \mathrm{ha}^{-1}\right)$ in Goiás semi-deciduous forests.

Thus, the greater representativeness of savanna phytophysiognomies in the fragments at an earlier stage of regeneration also gave rise to the differences and to species diversity, increasing the values of the latter to numbers closer to those proposed by Sposito \& Stehmann (2006). Another source of biological variability can be the conjugation of the permanence of species related to the beginning of succession and of the recruitment of species from more advanced stages, in a facilitation model (Connell \& Slatyer 1977), thus weakening possible dominance relationships among species. The occasional occurrence of disturbances can generate and/or maintain plant community diversity (Connell 1978) and prevent competitive exclusion. However, the increase in frequency and intensity of these disturbances impoverishes the community composition and structure.

During the Quaternary climatic fluctuations, oscillations in the territorial extension of biomes (Ledru 1993; Ledru et al. 1996; Ledru et al. 1998; OliveiraFilho \& Ratter 2000; Ab’sáber 2003; Joly 2007; Ledru et al. 2009;) provoked speciations and promoted geographical dissimilarities (Joly 2007; Ledru et al. 2009). Cerrado expanded over mesophytic forests, which were unable to re-expand their zone, a failure that can be attributed to human actions (Oliveira-Filho \& Ratter 2000). The succession process encompasses a recovery of species richness and diversity, guild recomposition, flora restoration and, finally, a vegetation restructuring (Brown \& Lugo 1990; Tabarelli \& Mantovani 1999; Oliveira-Filho etal. 2004). The Fazenda São Fernando in southern Goiás represents an ecotonal area between the Atlantic and Cerrado domains. Its vegetation was degraded and is currently undergoing a natural regeneration process. Characterizing the local vegetation and following its transformations can provide information allowing to understand the phytogeographical relationships. Although we found species richness and diversity values compatible with other semi-deciduous formations, the composition of the inventoried remnants showed floristic relationships closer to savanna tree communities. In Itumbiara, it was estimated that the Atlantic forest covered more than $50 \%$ of the township (SOS Mata Atlântica \& INPE 2008) and it may have been predominant in the study area. Thus, two hypotheses can be formulated: the past vegetation was mainly constituted by species 
of the Atlantic Forest, but environmental degradation drastically reduced their populations, and natural regeneration encompassed the recruitment of species from adjacent savanna physiognomies, in which case the balance between forest physiognomies and types would result from the interaction between disturbance frequency and intensity (Durigan \& Ratter 2006); or, the floristic relationships with Cerrado used to be and still are narrower, so that phytophysiognomy distinction depends mainly on edaphic attributes (Oliveira-Filho \& Ratter 2002) and the disturbance history affects the similarity between local communities because it impacts the succession process. Further clarification with regard to these hypotheses can come out of edaphic, paleoecological and palynological surveys or even of a monitoring of the forest community development over time (Durigan \& Ratter 2006). This scenario demonstrates how difficult it is to delimit zones or vegetation types. Nevertheless, the forest community inventoried in Itumbiara, Goiás, presented high species richness and it is located in a transitional area between two hotspots characterized by the urgency of surveys describing their biological diversity and allowing to implement conservationist activities.

\section{References}

Ab’Sáber, A.N. 2003. Os domínios de natureza no Brasil: potencialidades paisagísticas. Ateliê Editorial, São Paulo. 160p.

APG - Angiosperm Phylogeny Group III. 2009. An update of the Angiosperm Phylogeny Group classification for the orders and families of flowering plants: APG III. Botanical Journal of the Linnaean Society 161: 105-121.

Ayres, M.; Ayres Júnior. M.; Ayres, D.L. \& Santos, A.A.S. 2007. BioEstat: aplicações estatísticas nas áreas das ciências bio-médicas. Instituto de Desenvolvimento Sustentável Mamirauá, Belém. 364p.

Balduino, A.P.C.; Souza, A.L.; Meira-Neto, J.A.A.; Silva, A.F. \& Silva Júnior, M.C. 2005. Fitossociologia e análise comparativa da composição florística do Cerrado da flora de Paraopeba-MG. Revista Árvore 29: 25-34.

Braak, C.J.F. ter. 1995. Ordination. In: Jongman, R.H.G.; Braak, C.J.F.ter \& Tongeren, O.F.R. van. Data analysis in community and landscape ecology. Cambridge University Press, Cambridge. Pp. 91-173

Brower, J.E. \& Zar, J.H. 1984. Field \& laboratory methods for general ecology. Wm. C. Brown Publishers, Iowa. 226p.

Brown, S. \& Lugo, A.E. 1990. Tropical secondary forests. Journal of Tropical Ecology 6: 1-32.
Carvalho, A.R. \& Marques-Alves, S. 2008. Diversidade e índice sucessional de uma vegetação de Cerrado sensu stricto na Universidade Estadual de Goiás-UEG, campus de Anápolis. Revista Árvore 32: 81-90.

Castellani, T.T. \& Stubblebine, W.H. 1993. Sucessão secundária inicial em mata tropical mesófila, após perturbação por fogo. Revista Brasileira de Botânica 16: 181-203.

Colwell, R.K. 2006. EstimateS: Statistical estimation of species richness and shared species from samples, Version 8. Persistent URL purl.oclc.org/estimates.

Connell, J.H. 1978. Diversity in tropical Rainforest and coral reefs. Science 199: 1302-1310.

Connell, J.H. \& Slatyer, R.O. 1977. Mechanisms of succession in natural communities and their role in community stability and organization. The American Naturalist 111: 1119-1140.

Costa, A.A. \& Araújo, G.M. 2001. Comparação da vegetação arbórea de Cerradão e de Cerrado na Reserva do Panga, Uberlândia, Minas Gerais. Acta botanica brasilica 15: 63-72.

Durigan, G. \& Ratter, J.A. 2006. Successional changes in Cerrado and Cerrado/Forest ecotonal vegetation in Western São Paulo State, Brazil, 1962-2000. Edinburgh Journal of Botany 63: 119-130.

EPE - Empresa de Pesquisa Energética. 2006. Avaliação ambiental integrada dos aproveitamentos hidrelétricos da bacia do rio Paranaíba. Ministério de Minas e Energia, Brasília. 466p.

Espírito-Santo, F.D.B.; Oliveira-Filho, A.T; Machado, E.L.M.; Souza, J.S.; Fontes, M.A.L. \& Marques, J.J.G.S.M. 2002. Variáveis ambientais e a distribuição de espécies arbóreas em um remanescente de floresta estacional semidecídua montana no campus da Universidade Federal de Lavras, MG. Acta Botanica Brasilica 16: 331-356.

Felfili, J.M. 2003. Fragmentos de florestas estacionais do Brasil central: diagnóstico e propostas de corredores ecológicos. In: Costa, R.B. Fragmentação florestal e alternativas de desenvolvimento rural na região Centro-Oeste. UCDB, Campo Grande. Pp. 139-160.

Fonseca, M.S. \& Silva Júnior. M.C. 2004. Fitossociologia e similaridade florística entre trechos de Cerrado sentido restrito em interflúvio e em vale no Jardim Botânico de Brasília, DF. Acta botanica brasilica 18: 19-29.

Frelich, L.E.; Sugita, S.; Reich, P.B.; Davis, M.B. \& Friedman, S.K. 1998. Neighbourhood effects in forests: implications for within-stand patch structure. Journal of Ecology 86: 149-161.

Haidar, R. F.; Felfili, J. M.; Pinto, J. R. R. \& Fagg. C. W. 2005. Fitossociologia da vegetação arbórea em fragmentos de floresta estacional, no Parque Ecológico Altamiro de Moura Pacheco, GO. Boletim do Herbário Ezechias Paulo Heringer 15: 19-46.

Hill, J.L. \& Curran, P.J. 2003. Area, shape and isolation of tropical forest fragments: effects on tree species 
diversity and implications for conservation. Journal of Biogeography 30: 1391-1403.

Imaña-Encinas, J.; Macedo, L. A. \& Paula, J. E. 2007. Florística e fitossociologia de um trecho da floresta estacional semidecidual na área do Ecomuseu do Cerrado, em Pirenópolis-Goiás. Cerne 13: 308-320.

Joly, C.A. 2007. Biodiversidade e mudanças climáticas: contexto evolutivo, histórico e político. Ambiente e Sociedade X: 169-172.

Ledru, M. P. 1993. Late Quaternary environmental and climatic changes in central Brazil. Quaternary Research 39: 90-98.

Ledru, M.P.; Braga, P.I.S.; Soubiès, F.; Fournier, M.; Martin, L.; Suguio, K. \& Turcq, B. 1996. The last 50,000 years in the Neotropics (southern Brazil): evolution of vegetation and climate. Palaeogeography, Palaeoclimatology, Palaeoecology 123: 239-257.

Ledru, M.P.; Mourguiat, P. \& Riccomini, C. 2009. Related changes in biodiversity, insolation and climate in Atlantic rainforest since de last interglacial. Palaeogeography, palaeoclimatology, Palaeoecology 271: 140-152.

Ledru, M.P.; Salgado-Labouriau, M.L. \& Lorscheitter, M.L. 1998. Vegetation dynamics in southern and central Brazil during the last 10,000 yr B.P. Review of Palaeobotany and Palynology 99: 131-142.

Leitão Filho, H.F. 1987. Considerações sobre a florística de florestas tropicais e sub-tropicais do Brasil. Instituto de Pesquisas e Estudos Florestais 35: 41-46.

Lenoir, J.; Gégout, J.C.; Marquet, P.A.; Ruffray, P. de \& Brisse, H. 2008. A significant upward shift in plant species optimum elevation during the 20th century. Science 320: 1768-1771.

Malhi, Y.; Roberts, J.T.; Betts, R.A.; Killeen, T.J.; Li, W. \& Nobre, C.A. 2008. Climate change, deforestation, and the fate of the Amazon. Science 319: 169-172.

Mantgem, P. van; Stephenson, N.L.; Byrne, J.C.; Daniels, L.D.; Franklin, J.F.; Fulé, P.Z.; Harmon, M.E.; Larson, A.J.; Smith, J.M.; Taylor, A.H. \& Veblen, T.T. 2009. Widespread increase of tree mortality rates in the western United States. Science 323: 521-524.

Martins, F.R. 1991. Estrutura de uma floresta mesófila. Ed. UNICAMP, Campinas. 246p.

McCune, B. \& Mefford, M.J. 1999. PC-ORD 4.0: multivariate analysis of ecological data, user guide. MjM Software Design, Oregon.

Meira-Neto, J.A.A. \& Martins, F.R. 2002. Composição florística de uma floresta estacional semidecidual montana no município de Viçosa-MG. Revista Árvore 26: 437-446.

Mendonça, R.C.; Felfili, J.M.; Walter, B.M.; Silva Júnior., M.C.; Rezende, A.V.; Filgueiras, T.S. \& Nogueira, P.E. 1998. Flora vascular do bioma Cerrado. In: Sano, S.M. \& Almeida, S.P. Cerrado: ambiente e flora. EMBRAPA Cerrado, Planaltina. Pp. 287-556.
MMA - Ministério do Meio Ambiente. 2000. Avaliações e ações prioritárias para a conservação da biodiversidade da Mata Atlântica e Campos Sulinos. MMA/SBF, Brasília. 40p.

Mueller-Dombois, D. \& Ellenberg, H. 1974. Aims and Methods of Vegetation Ecology. John Wiley \& Sons, New York. 547p.

Nascimento, A.R.T.; Felfili, J.M. \& Meirelles, E.M. 2004. Florística e estrutura da comunidade arbórea de um remanescente de floresta estacional decidual de encosta, Monte Alegre, Goiás, Brasil. Acta Botanica Brasilica 18: 659-669.

Oliveira-Filho, A.T. 2006. Catálogo das árvores nativas de Minas Gerais: mapeamento e inventário da flora nativa e dos reflorestamentos de Minas Gerais. Editora UFLA, Lavras. 423p.

Oliveira-Filho, A.T. 2009. Treeatlan 1.0: Flora arbórea da Mata Atlântica e domínios adjacentes - um banco de dados envolvendo geografia, diversidade e conservação. Available in <http:// http://www.icb. ufmg.br/treeatlan>. Accessed 7 Dec 2009.

Oliveira-Filho, A.T.; Carvalho, D.A.; Vilela, E.A.; Curi, N. \& Fontes, M.A.L. 2004. Diversity and structure of the tree community of a fragment of tropical secondary forest of the Brazilian Atlantic Forest domain 15 and 40 years after logging. Revista Brasileira de Botânica 27: 685-701.

Oliveira-Filho, A.T.; Curi, N.; Vilela, E.A. \& Carvalho, D.A. 2001. Variation in tree community composition and structure with changes in soil properties within a fragment of semideciduous forest in south-eastern Brazil. Edinburgh Journal of Botany 58: 139-158.

Oliveira-Filho, A.T. \& Fontes, M.A.L. 2000. Patterns of floristic differentiation among Atlantic Forest in southeastern Brazil and the influence of climate. Biotropica 32: 793-810.

Oliveira-Filho, A.T. \& Ratter, J.A. 2000. Padrões florísticos das matas ciliares da região dos cerrados e a evolução das paisagens do Brasil central durante o Quaternário tardio. In: Rodrigues, R.R. \& Leitão Filho, H.F. Matas ciliares: bases multidisciplinares para estudo, conservação e restauração. EDUSP, São Paulo. Pp. 73-89.

Oliveira-Filho, A.T. \& Ratter, J.A. 2002. Vegetation physiognomies and woody flora of the Cerrado Biome. In: Oliveira, P.S. \& Marquis, R.J. The Cerrados of Brazil: ecology and natural history of a Neotropical savanna. Columbia University Press, New York. Pp. 91-120.

Ratter, J.A.; Bridgewater, S \& Ribeiro, J.F. 2003. Analysis of floristic composition of Brazilian Cerrado vegetation III: comparison of the woody vegetation of 376 areas. Edinburgh Journal of Botany 60: 57-109.

Ribeiro, J.F. \& Walter, B.M.T. 1998. Fitofisionomias do bioma cerrado. In: Sano, S.M. \& Almeida, S.P. Cerrado: ambiente e flora. EMBRAPA Cerrado, Planaltina. Pp. 89-166. 
Ribeiro, J.F.; Sano, S.M.; Macedo, J.; Silva, J.A. 1983. Os principais tipos fisionômicos da região dos cerrados. Boletim de Pesquisas 21. EMBRAPA Cerrado, Planaltina.

Rizzini, C.T. 1979. Tratado de Fitogeografia do Brasil aspectos sociológicos e florísticos. Hucitec, São Paulo. 374p.

Roberts, M.R. \& Gillian, F.S. 1995. Patterns and mechanisms of plant diversity in forested ecosystems: implications for forest management. Ecological Applications 5: 969-977.

Rosa, A.G. \& Schiavini, I. 2006. Estrutura da comunidade arbórea em um remanescente florestal urbano (Parque do Sabiá, Uberlândia, MG). Bioscience Journal 22: 151-162.

S.O.S. Mata Atlântica \& INPE - Instituto Nacional de Pesquisas Espaciais. 2008. Atlas dos remanescentes florestais da Mata Atlântica Período 2000-2005. Fundação SOS Mata Atlântica \& INPE, São Paulo. 157p.

Sabbag, S. C. 2003. Desmatamento. Informativo técnico. IBAMA/MMA, Brasília.

Salis, S.M.; Silva, M.P.; Mattos, P.P.; Silva, J.S.V.; Pott, V.J. \& Pott, A. 2004. Fitossociologia de remanescentes de floresta estacional decidual em Corumbá, estado do Mato Grosso do Sul, Brasil. Revista Brasileira de Botânica 27: 671-684.

SEPLAN-GO-Secretaria de Planejamento e Desenvolvimento do Estado de Goiás. 2005. Anuário estatístico do estado de Goiás-2005. SEPLAN, Goiânia. 826p.

Silva, J.M.C. \& Bates, J.M. 2002. Biogeographic patterns and conservation in the South America Cerrado: a tropical savanna hotspot. BioScience 52: 225-233.
Silva, L.A. \& Scariot, A. 2004. Comunidade arbórea de uma floresta estacional decídua sobre afloramento calcário na bacia do Paraná. Revista Árvore 28: 61-67.

Souza, J.P.; Araújo, G.M. \& Haridasan, M. 2007. Influence of soil fertility on the distribution of tree species in a deciduous forest in the Triângulo Mineiro region of Brazil. Plant Ecology 191: 253-263.

Souza, J.P.; Araújo, G.M.; Schiavini, I. \& Duarte, P.C. 2006. Comparation between canopy trees and arboreal lower strata of urban semideciduous seasonal forest in Araguari-MG. Brazilian Archives of Biology and Technology 49: 775-783.

Sposito, T. C. \& Stehmann, J. R. 2006. Heterogeneidade florística e estrutural de remanescentes florestais da Área de Proteção Ambiental ao sul da região metropolitana de Belo Horizonte (APA SulRMBH), Minas Gerais, Brasil. Acta Botanica Brasilica 20: 347-362.

Tabarelli, M.; Cardoso, J. M. \& Gascon, C. 2004. Forest fragmentation synergisms and the impoverishment of neotropical forests. Biodiversity and Conservation 13: 1419-1425.

Tabarelli, M. \& Mantovani, W. 1999. A regeneração de uma Floresta Tropical Montana após corte e queima (São Paulo-Brasil). Revista Brasileira de Biologia 59: 239-250

Tropicos.org. 2010. Missouri Botanical Garden. Available in <http://www.tropicos.org>. Accessed 25 Nov 2009.

Whittaker, R.H. 1972. Evolution and measurement of species diversity. Taxon 22: 213-251.

Zar, J.H.; 1999. Biostatistical analysis. Prentice-Hall, New Jersey. 929p. 